January 2010

\title{
Validation of c-reactive protein in the early diagnosis of neonatal sepsis in a tertiary care hospital in Kenya.
}

\author{
Rashim Kumar \\ Gertrude's Children's Hospital \\ Rachel Musoke \\ University of Nairobi \\ William Macharia \\ Aga Khan University, macharai.william@aku.edu \\ Gunturu Revathi \\ Aga Khan University, gunturu.revathi@aku.edu
}

Follow this and additional works at: http://ecommons.aku.edu/ eastafrica_fhs_mc_paediatr_child_health

Part of the Pediatrics Commons

\section{Recommended Citation}

Kumar, R., Musoke, R., Macharia, W., Revathi, G. (2010). Validation of c-reactive protein in the early diagnosis of neonatal sepsis in a tertiary care hospital in Kenya.. East African Medical Journal, 87(6), 255-261.

Available at: http://ecommons.aku.edu/eastafrica_fhs_mc_paediatr_child_health/11 
East African Medical Journal Vol. 87 No. 6 June 2010

VALIDATION OF C-REACTIVE PROTEIN IN THE EARLY DIAGNOSIS OF NEONATAL SEPSIS IN A TERTIARY CARE HOSPITAL IN KENYA

R. Kumar, MBChB, MMed, Consultant Paediatrician, Gertrude's Children's Hospital, P.O. Box 42325 - 00100, Nairobi, Kenya, R. Musoke, MBChB, MMed, Associate Professor and Neonatologist, Department of Paediatrics and Child Health, College of Health Sciences, University of Nairobi, P.O. Box 19676 - 00100, Nairobi, Kenya, W. M. Macharia, MBChB, MMed, Chairman and Head of Paediatric Department and G. Revathi, MD, Associate Professor of Microbiology, Aga Khan University Hospital, Nairobi, P.O. Box 30270 - 00100, Nairobi, Kenya

Request for reprints to: Dr. R. Kumar, Gertrude's Children's Hospital, P.O. Box 42325 - 00100, Nairobi, Kenya

\title{
VALIDATION OF C-REACTIVE PROTEIN IN THE EARLY DIAGNOSIS OF NEONATAL SEPSIS IN A TERTIARY CARE HOSPITAL IN KENYA
}

\author{
R. KUMAR, R. MUSOKE, W. M. MACHARIA and G. REVATHI
}

\begin{abstract}
Objective: To evaluate utility of C-reactive protein (CRP) in the early diagnosis of neonatal sepsis in a tertiary care Newborn Unit in Kenya.

Design: Cross-sectional study.

Setting: Newborn Unit, Kenyatta National Hospital.

Subjects: All neonates admitted to Newborn Unit, Kenyatta National Hospital during the study period with suspected sepsis based on specified clinical criteria.

Results: Of the 310 infants, there were 83 episodes of proven sepsis and 94 episodes of probable sepsis. Using the standard CRP cut-off value of $5 \mathrm{mg} / \mathrm{dl}$, a sensitivity of $95.2 \%$ in proven sepsis and $98.9 \%$ for probable septic episodes were noted. In proven sepsis, a specificity of $85.3 \%$, positive predictive value of $80.6 \%$, and a negative predictive value of $96.5 \%$ were noted. In probable sepsis, a specificity of $83.3 \%$, positive predictive value of $80.9 \%$ and a negative predictive value of $99.1 \%$ were noted. The overall accuracy in proven sepsis was $96.5 \%$, and in probable sepsis was noted to be $99.1 \%$. Sub-analysis showed a lower positive predictive value $(61.5 \%)$ for early onset sepsis compared to 93\% for late onset sepsis. Repeat CRP tests were done in 33 babies. Twenty two of the 29 with proven/probable infection had a ten-fold increase in CRP levels, butlevels were noted to be low or reducing in seven $(24.1 \%)$ babies showing signs of improvement clinically. Using a receiver operator characteristic curve, the optimal cut-off point for CRP was found to be $5 \mathrm{mg} / \mathrm{dl}$.

Conclusions: Serum CRP is an accurate indicator of neonatal sepsis, with high sensitivity, specificity and predictive values, at the standard cut-off of 5. CRP is a better screening test for late-onset than early-onset neonatal sepsis. The standard recommended CRP cut -off point of 5 is appropriate for local use.
\end{abstract}

\section{INTRODUCTION}

Neonatal sepsis is a clinical syndrome characterised by a rapid onset of systemic signs and a high morbidity and mortality (1). In Kenya, Musoke etal (2-4) reported a high sepsis rate with a case fatality rate of $20-42 \%$. Early diagnosis and treatment are essential but subtle and non-specific clinical features and unavailability of rapid, accurate and cost-effective laboratory tests have made this difficult. Blood culture which is the gold standard takes 48-72 hours and in many septic infants it may be negative $(1,5)$.

Normally, there is no CRP in blood serum. CRP is secreted in increased amounts within six hours of acute inflammatory stimulus, and continues toincrease several hundred-fold within 24-48 hours. After effective treatment, levels fall rapidly within five to seven hours. Persistently elevated CRP during antibiotic therapy should suggest the possibility of fungal infection, resistant organisms or development of a complication (6-8). Several workers have evaluated this test and reported high sensitivity and specificity. It is probably the one test that helps in diagnosing sepsis in infants with negative blood cultures (8-11). CRP levels can be elevated in conditions such as meconium aspiration, perinatal asphyxia and tissue injury. Although a result above $1 \mathrm{mg} /$ dlis usually considered high for CRP, most infections and inflammations result in CRP levels above $10 \mathrm{mg} / \mathrm{dl}$. Universally, a cut-off level of $5 \mathrm{mg} / \mathrm{dl}$ has been accepted as the best predictor of neonatal sepsis, with a maximum sensitivity, specificity and negative predictive value $(8,12)$.

CRP assays are not a routine test yet in government run institutions in Kenya. This study was aimed at evaluating the usefulness of CRP as a screening test in the early diagnosis of sepsis in neonates admitted to Kenyatta National Hospital $(\mathrm{KNH})$ with suspected sepsis. 


\section{MATERIALS AND METHODS}

A cross-sectional study was carried out in the Newborn Unit of KNH, the national tertiary referral and teaching hospital.

Study population and case definition: All neonates admitted to KNH Newborn Unit during the study period with suspected sepsis based on perinatal risk factors or suspicious clinical findings were recruited before first-line (benzyl penicillin and gentamicin) or change over to second-line antibiotics (second or third generation cephalosporin and amikacin) were initiated. Neonates whose parents/guardians declined to give consent and those with history of meconium aspiration, perinatal asphyxia, tissue injury and severe hepatocellular involvement were excluded. Neonates were classified into proven sepsis (culture positive), probable sepsis (culture negative but clinical haematological features suggestive), no sepsis (clinical features subsided within 12 hours, microbiological/ haematological features not suggestive). The diagnosis at each evaluation was categorised without consideration of CRP levels.

The required sample size was 276 using the appropriate statistical formula for a descriptive crosssectional study. Proportion of total population with positive culture was taken to be $30 \%$ based on thelocal estimates of $6-60 \%$. As accuracy of the study could not be determined from other studies, sensitivity of $98 \%$ was used to calculate the sample size. The level of statistical significance was set at 0.03 as was the margin of precision error.

To control for subjectivity, neonates were included into the study, based on an agreement in the clinical findings between attending clinician and investigator.

Laboratory methods: A total of $1.5 \mathrm{mls}$ of blood was drawn from each infant for complete blood count

Table 1

Baseline characteristics of study population associated with patient category

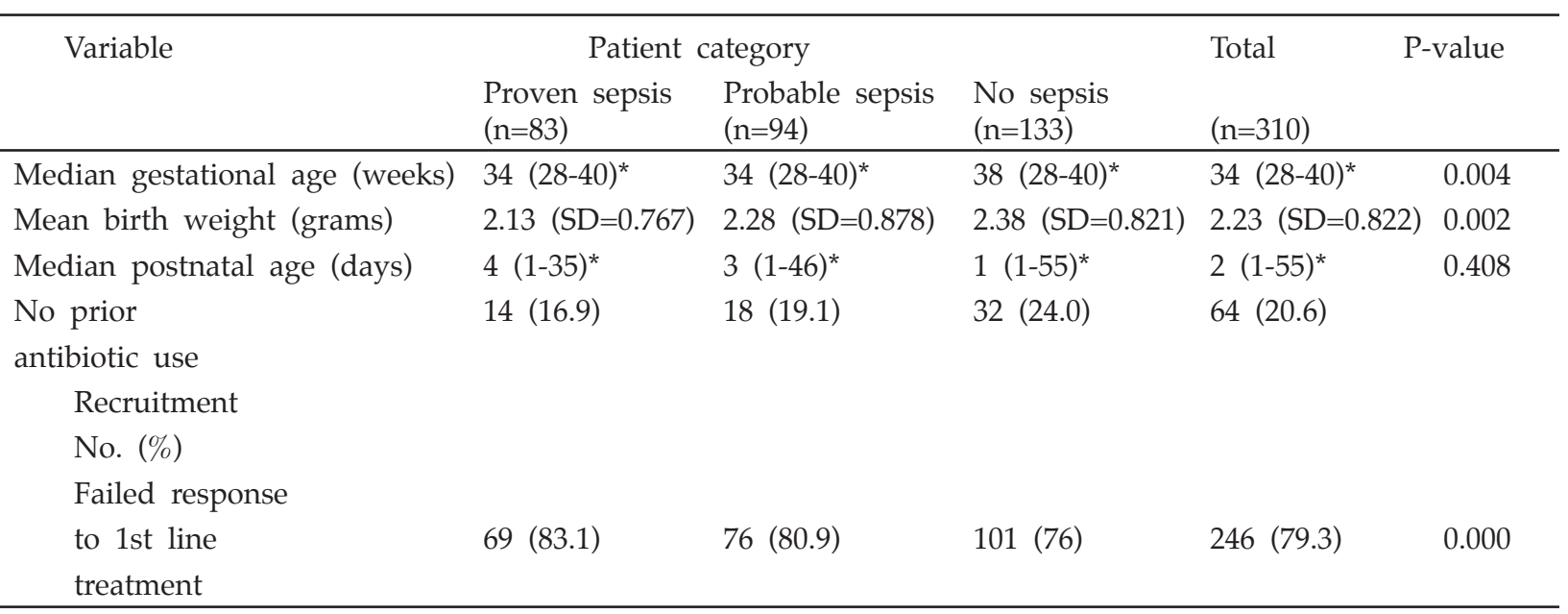

(CBC), culture and CRP assays. CBC and culture were done using standard procedures in haematology and microbiology laboratories. CSF, stool and urine tests, wherever indicated, was collected and processed by standard laboratory techniques.

Radiological studies were done as indicated: Samples for CRP were stored at $-20^{\circ} \mathrm{C}$ and analysed as a batch. The test principle was immuno-turbidimetric assay. Measuring range: $0.3-24 \mathrm{mg} / \mathrm{dl}(0.003-0.24 \mathrm{~g} / \mathrm{l})$ Using Rodwell's haematological scoring system, a score of three or more, identified infants with sepsis.

Data management and statistical analysis: All data from this study were analysed using SPSS software version 12.0. Receiver-operator characteristic (ROC) curve was constructed to permit selection of threshold values. Based on this cut-off point, the diagnostic utility of CRP was determined using accuracy, sensitivity, specificity and predictive values.

Ethical considerations: Study was undertaken after approval by the Department of Paediatrics and Child Health, University of Nairobi, and the Ethics and Review Committee, KNH. Informed written consent was also obtained from the parents/guardians of the neonates recruited into the study following explanation of the study protocol.

\section{RESULTS}

A total of 310 patients satisfied the inclusion criteria and were recruited over a three month period (JuneSeptember 2005).

Baseline and clinical characteristics: Of the 310 infants recruited, the male: female ratio was 1:1, with no significant difference in the number of term and preterm neonates in the study group $(\mathrm{P}=0.062)$. Factors associated with sepsis occurred with similar frequency in both terms and preterms.

* Range shown in brackets, SD = Standard Deviation 
As shown in Table 1, when compared with infants with no sepsis, those with sepsis had a significantly lower mean birth weight $(\mathrm{P}$-value $=0.002)$ and mediangestationalage $(\mathrm{P}$-value $=0.004)$. Asignificant difference $(P$-value $=0.000)$ was noted in the sepsis rate among neonates recruited before first-line and second-line antibiotics, with proven sepsis being more frequent in late-onsetepisodes (attack rate 40\%) compared to early-onset episodes (attack rate 16\%). Probable sepsis was diagnosed in $21 \%$ of early-onset episodes and $42 \%$ of late-onset episodes.
Correlation ofdiagnosis and serum CRP levels: CRPvalues were compared in septic, suspected septic and nonseptic groups at the standard cut-off of $5 \mathrm{mg} / 1$. Test was considered to be positive when CRP was $>5 \mathrm{mg} / 1$. Proven or probable sepsis was strongly correlated with elevated CRP levels. The calculated sensitivities and specificities of each testing strategy are shown in the Tables 3 and 4 . To assess the ability of abnormal and normal CRP levels to identify the presence or absence of infection, respectively, the positive and

Table 2

Frequency distribution of aetiological organisms cultured from blood and CSF

\begin{tabular}{llllll}
\hline Gram-negative organism & $(\%)$ & Gram-positive organism & $(\%)$ & Fungus & $(\%)$ \\
\hline Enterobacter agglomerans & 26.5 & Coagulase negative staph. & 13.4 & Candida & 4.5 \\
Citrobacter & 12.3 & Staphylococcus aureus & 4.5 & \\
Klebsiella pneumonia & 13.4 & Enterococcus & 4.5 & \\
Acinetobacter spp & 7.8 & & & \\
Escherichia coli & 6.7 & & & \\
Unidentified gram-negative bacilli & 4.5 & & & \\
Salmonella typhi & 1.1 & & & \\
\hline
\end{tabular}

Overall in-hospital mortality was higher in patients with sepsis (definite and probable), compared withno sepsis. Sepsis rate was noted to be predominant in neonates older than two days. There was no significant difference in the rate of sepsis among males and females in the different postnatal groups.

Organisms were isolated from 83 blood cultures and two CSF cultures. As shown in Table 2, overall $72.7 \%$ were gram negative organisms, $22.4 \%$ were gram positive organisms and $4.5 \%$ of the isolates were Candida. negative predictive values for each testing strategy were calculated.

A high sensitivity of $95.2 \%$ in proven septic episodes and $98.9 \%$ for probable septic episodes were noted. In proven sepsis, a specificity of $85.3 \%$, a PPV of $80.6 \%$ and a NPV of $96.5 \%$ were noted. In probable infection, a specificity of $83.3 \%$, PPV of $80.9 \%$ and a NPV of $99.1 \%$ were noted. The overall accuracy in proven sepsis was $96.5 \%$, and in probable sepsis was noted to be $99.1 \%$ (Tables 3 and 4).

Table 3

Diagnostic utility of C-Reactive protein in babies with proven sepsis

\begin{tabular}{lllc}
\hline Test result & Proven sepsis & No sepsis & Total \\
\hline Positive & 79 & 19 & 98 \\
Negative & 4 & 110 & 114 \\
\hline Total & 83 & 129 & 212 \\
\hline
\end{tabular}

Sensitivity $=79 / 83=95.2 \%$

Specificity $=110 / 129=85.3 \%$

Positive predictive value $=79 / 98=80.6 \%$

Negative predictive value $=110 / 114=96.5 \%$

Overall accuracy $=189 / 212=89.1 \%$ 
Table 4

Diagnostic utility of C-Reactive protein in babies with probable sepsis

\begin{tabular}{lccc}
\hline Test result & $\begin{array}{c}\text { Probable } \\
\text { sepsis }\end{array}$ & No sepsis & Total \\
\hline Positive & 93 & 22 & 115 \\
Negative & 1 & 110 & 111 \\
\hline Total & 94 & 132 & 226 \\
\hline
\end{tabular}

Sensitivity $=93 / 94=98.9 \%$

Specificity $=110 / 132=83.3 \%$

Positive predictive value $=93 / 115=80.9 \%$

Negative predictive value $=110 / 111=99.1 \%$

Overall accuracy $=203 / 226=89.8 \%$

As shown in Tables 5-8, a further sub-analysis was done in the study patients, classifying them into early-onset and late-onset septic categories. Serum CRP levels were noted to be $>5 \mathrm{mg} / 1$ in babies with proven infection, $88.9 \%$ in early and $98.2 \%$ in lateonsetepisodes. In probableinfection, high CRPlevels were noted in all patients in the early-onset and 98.2\% patients in the late-onset episodes. Sensitivity and specificity of CRP measurements for late-onset proven infections was higher than for early-onset proven episodes. The positive predictive value was low in early onset episodes, compared to the late onset episodes where the positive predictive values were more than $93 \%$. Negative predictive value was high in both early and late-onset episodes. Overall accuracy was higher in late-onset than in early-onset episodes.

Early-onset episodes:

Table 5

Diagnostic utility of C-Reactive protein in babies with proven sepsis

\begin{tabular}{lccc}
\hline Test result & $\begin{array}{c}\text { Proven } \\
\text { sepsis }\end{array}$ & No sepsis & Total \\
\hline Positive & 24 & 15 & 39 \\
Negative & 3 & 85 & 88 \\
\hline Total & 127 & 100 & 127 \\
\hline
\end{tabular}

Sensitivity $=24 / 27=88.9 \%$

Specificity $=85 / 100=85 \%$

Positive predictive value $=24 / 39=61.5 \%$

Negative predictive value $=85 / 88=96.6 \%$

Overall accuracy $=109 / 127=85.8 \%$
Table 6

Diagnostic utility of C-Reactive protein in babies with probable sepsis

\begin{tabular}{lccc}
\hline Test result & $\begin{array}{c}\text { Probable } \\
\text { sepsis }\end{array}$ & No sepsis & Total \\
\hline Positive & 37 & 18 & 55 \\
Negative & 0 & 85 & 85 \\
\hline Total & 37 & 103 & 140 \\
\hline
\end{tabular}

Sensitivity $=37 / 37=100 \%$

Specificity $=85 / 103=82.5 \%$

Positive predictive value $=37 / 55=67.3 \%$

Negative predictive value $=85 / 85=100 \%$

Overall accuracy $=122 / 140=87.1 \%$

Late-onset episodes

\section{Table 7}

Diagnostic utility of C-Reactive protein in babies with proven sepsis

\begin{tabular}{lccc}
\hline Test result & Proven sepsis & No sepsis & Total \\
\hline Positive & 55 & 4 & 59 \\
Negative & 1 & 25 & 26 \\
\hline Total & 56 & 29 & 85 \\
\hline
\end{tabular}

Sensitivity $=55 / 56=98.2 \%$

Specificity $=25 / 29=86.2 \%$

Positive predictive value $=55 / 59=93.2 \%$

Negative predictive value $=25 / 26=96.1 \%$

Overall accuracy $=80 / 85=94.1 \%$

Table 8

Diagnostic utility of C-Reactive protein in babies with probable sepsis

\begin{tabular}{|c|c|c|c|}
\hline Test result & Probable sepsis & No sepsis & Total \\
\hline Positive & 56 & 4 & 60 \\
\hline Negative & 1 & 25 & 26 \\
\hline Total & 57 & 29 & 86 \\
\hline
\end{tabular}

Sensitivity $=56 / 57=98.2 \%$

Specificity $=25 / 29=86.2 \%$

Positive predictive value $=56 / 60=93.3 \%$

Negative predictive value $=25 / 26=96.1 \%$

Overall accuracy $=81 / 86=94.2 \%$

Repeat CRP tests were done on 33 infants with suspected sepsis, 48 hours after the initial sampling; a ten fold increase in serum CRP levels was noted in 
babies with proven/probable infection compared to initial CRP values. Neonates with infections not responding to antibiotic treatment had a persistent rise in CRP levels. The second CRP levels were noted to be low in babies showing signs of improvement clinically. One infected patient in whom the CRP did not exceed the normal range showed a dramatic 200fold rise from $0.015 \mathrm{mg} / \mathrm{l}$ on day one, to $3.02 \mathrm{mg} / 1$ after 48 hours. Serum CRP levels remained more or less the same in the non-septic category (Figure 1).

Using the ROC curve, the point on the curve that lies closest to the upper left corner, best defines the cutoff that can be used in order to determine the utility of CRP as seen in the Figure 2. When computed with SPSS version 12.0, this corresponds to a CRP level of $5 \mathrm{mg} / \mathrm{dl}$, in-keeping with the standard recommended cut-off.

\section{Figure 1}

Comparison of baseline and 24- 48 hours CRP levels among antibiotic responders and non-responders

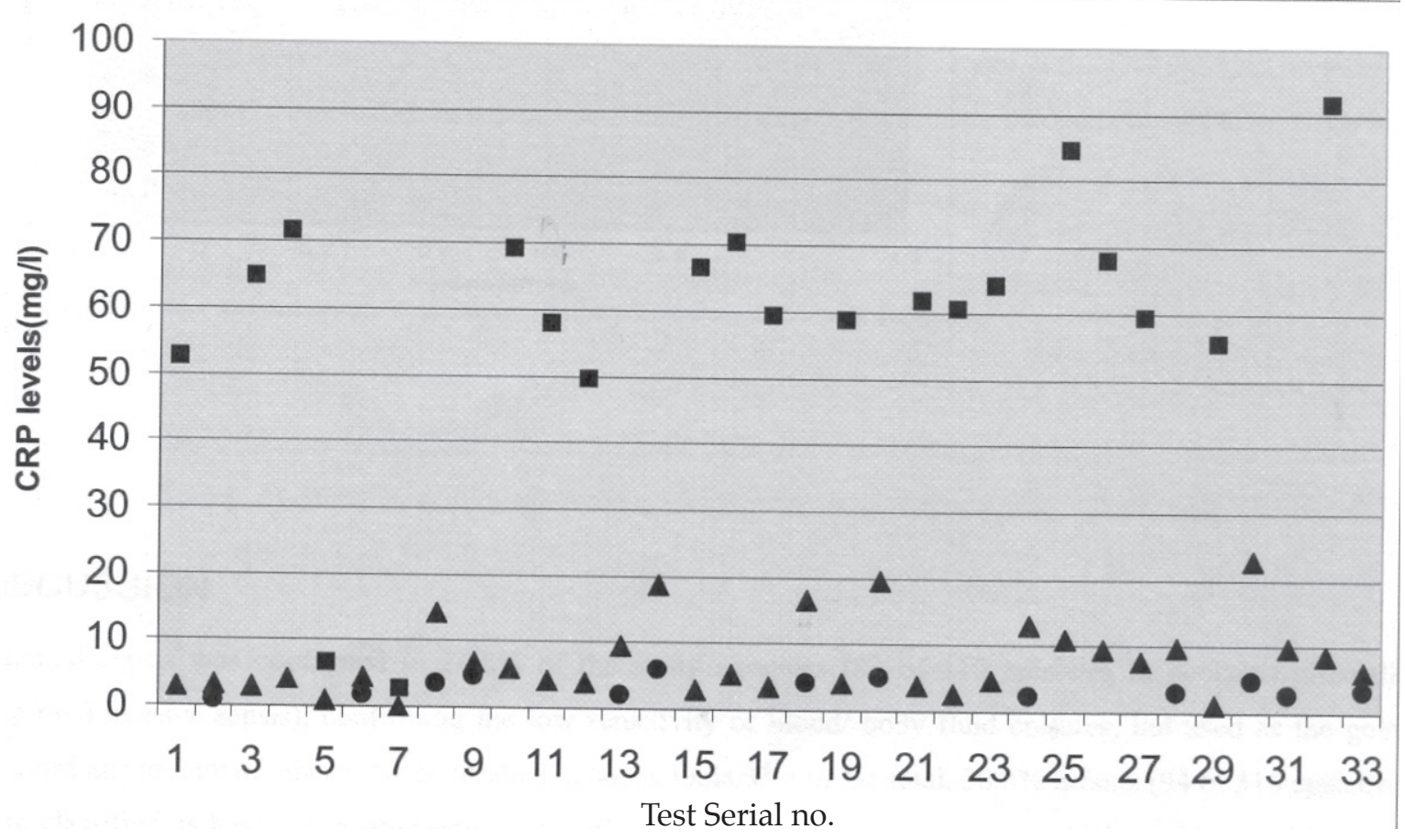

Figure 2

Receiver operating characteristic (ROC) curve for C-Reactive protein

ROC for probable and proven sepsis

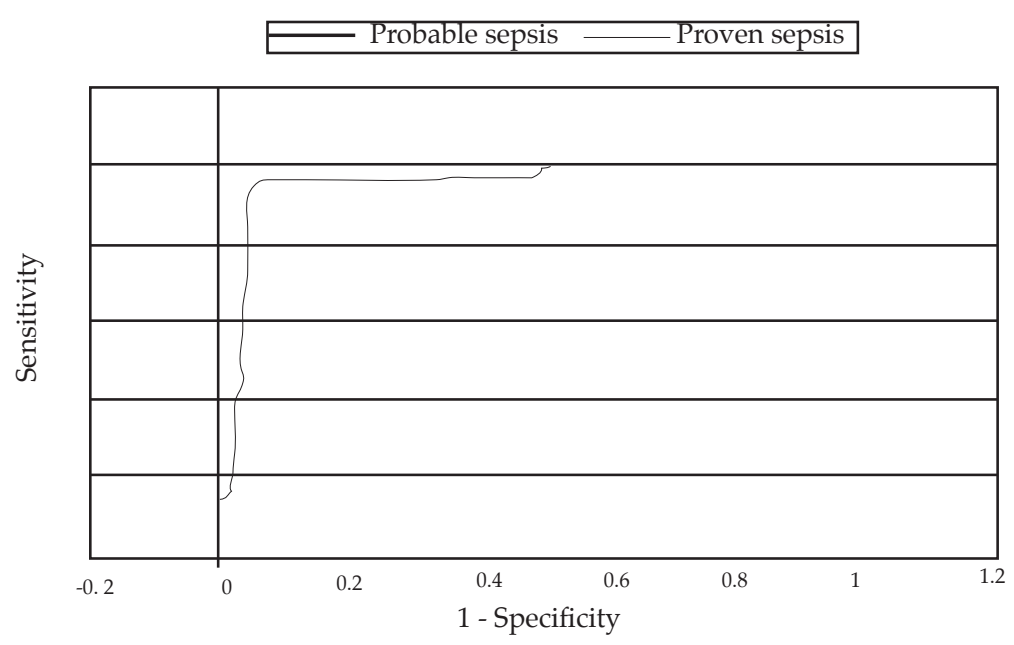




\section{DISCUSSION}

Bacterial sepsis was confirmed in $26.8 \%$ of the study neonates (83 of 310 episodes in neonates clinically suspected to have sepsis), confirming the low sensitivity of blood body fluid cultures, but used as the gold-standard due to lack of other more accurate diagnostic tools. Out of the total, 30.3\% infants (94 of 310 episodes) were classified as having probable sepsis, comprising a difficult diagnostic group which could not be ignored, as fatal infection has been reported in the presence of negative blood cultures. In the majority of these infants, intrapartum or postpartum antibiotic therapy probably influenced culture results (13).

Serum concentrations of CRP increase several hundredfold in response to infection, but recognition that a delay of at least six to eight hours is intrinsic to the cascade of events leading to elevation of serum CRP levels led to appropriate criticisms of this test as having insufficient sensitivity to guide therapy either by reliably diagnosing or excluding bacterial infection (14). Philip et al (15) suggested that serial normal levels are useful for identification of infants who do not have bacterial infection. Gerdes and Polin (9) reported high sensitivity (93\%) and NPV (99\%) for CRP levels determined by a latex agglutination method at the time of evaluation and 12 to 24 hours later. Pourcyrous and Boda (16) found that two levels measured over the first three days in neonates with infection had a high NPV, and suggested that the utility of CRP levels might be optimised by obtaining serial levels at 12-hour intervals in the first 24 to 36 hours of illness. Philip and Hewitt (17) reported no recurrence of infection within 7 days of discontinuation of antibiotics based on three normal CRP determinations within 48 hours and negative cultures, in low birth weight infants at risk for earlyonset infection. Based on these data, determinations of serum CRP levels were incorporated into our diagnostic approach to suspected neonatal sepsis.

The sensitivity of CRP was found to be $88.9 \%$ for early onset proven sepsis and $98.2 \%$ for late-onset proven sepsis. Despite this high sensitivity, one would have desired an even higher sensitivity for early-onset episodes such that very few or none of the cases would be missed in neonates with such a life-threatening illness. The lower scores for sensitivity in early-onset episodes reflect the lag period between the onset of infection and production of sufficient CRP to exceed the normal range. The higher prevalence of elevated CRP levels in late-onset episodes is not unexpected, because their infections had been present for sufficient time to produce persistent elevation in serum CRP levels. The study showed the CRP to have a specificity of $82.5 \%$ for early onset episodes and $86.2 \%$ for late onset episodes. The specificity of CRP was found to be lower than the $100 \%$ found by Wasunna et al
(18). The low specificity found in our study could be attributed to the influence of various non-infectious neonatal conditions (meconium aspiration, perinatal asphyxia, tissue injury). Studies done at KNH have shown unreliability of Apgar Scores for babies born at various health facilities (19); this could also have influenced our study and led to recruitment of babies with perinatal asphyxia. Many of these conditions, however, are predisposing factors for neonatal sepsis and therefore difficult to control for in such a study. Culture negative sepsis due to anaerobic organisms and viruses that were not tested for in our study may also have resulted in high false positive rate and therefore a low specificity.

Intrapartum maternal exposure to antimicrobial agents may also have contributed to the low specificity, by increasing the number of culture-negative but test-positive cases; this factor was not controlled for in the study. Neonates born to mothers who have received intrapartum antibiotics are still at risk for sepsis and need to be evaluated and treated for sepsis as appropriate, and this was done in this study. However, neonates were recruited into the study only before initiation of first-line or change to second-line therapy, in an attempt to minimise the influence of prior antimicrobial exposure on culture yield.

The CRP values had a positive predictive value of $61.5 \%$ in early-onset sepsis and $93.2 \%$ for late-onset sepsis. The lower scores for positive predictive values reflect the lag period between the onset of infection and production of sufficient CRP toexceed the normal range. The negative predictive values in this study were found to be $96.6 \%$ for early-onset sepsis and $96.1 \%$ for late-onset sepsis, which is reasonably high and consistent with the high sensitivity and is also in keeping with studies done elsewhere.

In attempting to diagnose a serious conditionsuch as neonatal sepsis, a condition that is life threatening and yet treatable, a diagnostic test with maximal sensitivity and specificity is desirable. Elevated CRP levels strongly correlated with infection for both early-onset and late-onset episodes, whether single or serial levels were considered and independent of whether probable cases were grouped with proven cases, or considered separately. The diagnosticutility of CRP was compared in early-onset and late-onset episodes. The overall accuracy was slightly higher for the diagnosis of late-onset sepsis than early-onset sepsis.

A receiver operating characteristic (ROC) curve was plotted to determine the best cut-off value for CRP. A cut-off value of five was found to give the best compromise between the true positive rate (sensitivity) and the false positive rate (1-specificity). In a life-threatening and rapidly progressive condition such as neonatal sepsis, the need for a very sensitive test justified the use of $5 \mathrm{mg} / \mathrm{dl}$ as the cut-off, inkeeping with standard universal cut-off. 
Is there any role of CRP in clinical-decision making? A major role for serum CRP measurement lies in its capacity to exclude the diagnosis of sepsis if CRP remains normal. The converse is also true and a raised CRP should greatly enhance the suspicion of infection. CRP can therefore be used to initiate treatment even in the absence of other evidence. CRP was shown to fall in response to effective antimicrobial therapy and a rapid, sensitive assay such as we have used should be valuable for objective monitoring of the success and duration of treatment.

Cost effectiveness was not part of the current study but in a study done elsewhere, the total costs of oral and parental antibiotics were compared between two groups: those subjected to advance testing (CRP and WBC counts at initial evaluation) and those who were not. A 30\% reduction in costs was achieved with advanced testing (20). In the current study, the cost of the tests was US dollars 2.7 per test. This cost is certainly much less than what would be spent in the treatment of a baby when one cannot exclude infection. In addition the test takes only 15 minutes and does not require professional expertise. Future development of a non-isotopic counterpart of the present assay should make the benefits of CRP testing in neonatal practice available as a side-room test in paediatric units generally.

The limitation of the study was that serial serum CRP assays were difficult to perform due to financial constraints for CRP assays.

In conclusions, serum CRP is an accurate indicator of neonatal sepsis. CRP is a better screening test for late-onset than early onset neonatal sepsis. The standard recommended CRP cut-off point of $5 \mathrm{mg} / 1$ is appropriate for local use. All babies with suspected sepsis should be screened. If initial level is low, repeat CRP test done after 8-12 hours will help in indicating which babies require antibiotic therapy.

\section{REFERENCES}

1. Klein, J.O. and Marcy, S.M. Bacterial sepsis and meningitis. In: Remington J.S. and Klein J.O. Eds. Infectious diseases of the fetus and newborn infant, 4th ed. U.S.A: WB Saunders. 1995; 835-878.

2. Musoke, R.N. and Malenga, G.J. Bacterial infections in neonates at $\mathrm{KNH}$ nursery: a prospective study. East Afr. Med. J. 1984; 61: 906-916.

3. Kasirye- Bainda, E. and Musoke, R.N. Neonatal morbidity and mortality at $\mathrm{KNH}$, Newborn Unit East Afr. Med. J. 1992; 69: 360-365.
4. Musoke, R.N. Emergence of multi drug resistant gram negative organisms in a neonatal unit and the therapeutic implications. J. Trop. Pediatr. 2000; 46: 36-41.

5. Baley, J.E. and Goldfarb, J. Neonatal infections. In: care of the high risk neonate. 4th ed. Klaus M.H., Fanaroff, A.A. WB Saunders. 1993; 323-331.

6. Freij, B.J. and McCracken, G.H. Acute Infections. In: Neonatology; Pathophysiology and management of the newborn. 4th ed. GB Avery, Fletcher MA, Macdonald MG. Lippincort- Raven. 1997; 1088-1089.

7. Beck, C.M. and Azimi, P. Bloodstream infections in neonatal intensive care unit patients: results of a multicenter study. Pediat Infect. Dis. J. 1994; 13: 11101116.

8. De Silva, O., Arne, O. and Kenyon, C. Accuracy of leukocyte indices and CRP for diagnosis of neonatal sepsis: a critical review. Pediatr Infec. Dis. J. 1995; 14: 362-366.

9. Gerdes, J.S. and Polin, R.A. Sepsis screen in neonates with evaluation of plasma fibronectin. Pediatr. Infect. Dis. J. 1987; 6: 443-446.

10. Benitz, W.E., Han, M.Y., Madanm A. and Ramachandra, P. Serial serum CRP levels in the diagnosis of neonatal infection. Pediatr. 1998; 102: 41-54.

11. Forest, J.C. and Lariviere, F. CRP as biochemical indicator of bacterial infection in neonates. Clin. Biochem. 1986; 19: 192-194.

12. Magny, J. F. and Benatur, C. CRP and the diagnosis of neonatal infection: Pediatr. 1986; 41: 105-108

13. Dacie, J.V. and Lewis, S.M. Practical Haematology, 7th ed. Churchill Livingstone. 1991; 1.

14. Seibert, K. and $\mathrm{Yu}, \mathrm{V}$. The value of CRP protein measurement in the diagnosis of neonatal infection. J. Pediatr. Child Hlth. 1990; 26: 267-270.

15. Philip, A.G.S. Response of CRP in neonatal group B streptococcal infection. Pediatr. infect. Dis. J. 1985; 4: 145-148.

16. Pourcyrous, M. and Bada, H.S. Acute phase reactants in neonatal bacterial infections. J. Perinatol. 1991; 11: 319-325.

17. Philip, A.G.S. and Hewitt, J.R. Early diagnosis of neonatal sepsis. Pediatrics. 1980; 65: 1036-1041.

18. Wasunna, A., Whitelaw, A., Gallimore, R. et al. $\mathrm{C}$-reactive protein and bacterial infection in preterm infants. Eur. J. Pediatr. 1990; 149: 424-427.

19. Coro, I. The accuracy of Apgar score assigned by health workers at Kenyatta National Hospital and Pumwani Maternity Hospital, Kenya. MMed. Thesis. 1992.

20. Takemura, Y., Ishida, H., Saitoh, H. et al. Economic consequence of immediate testing for C-reactive protein and leukocyte count in new outpatients with acute infection. Clin. Chimica. Acta. 2005; 360: 114-121. 